\title{
Robust Control of a Quadcopter Flying via Sliding Mode
}

\author{
Rudi Uswarman ${ }^{1}$, Swadexi Istiqphara ${ }^{1}$, Rajif Agung Yunmar ${ }^{2}$, Arkham Zahri \\ Rakhman $^{2}$ \\ ${ }^{1}$ Electrical Engineering Department, Institut Teknologi Sumatera, Lampung Selatan, Lampung \\ ${ }^{2}$ Informatics Engineering Department, Institut Teknologi Sumatera, Lampung Selatan, Lampung
}

\begin{abstract}
Sliding Mode Control (SMC) used to control the stability of a quadcopter from disturbances and uncertainties. This technique has two main advantages: the nonlinear dynamics and modelling errors of the quadcopter can be eliminated by switching function and the uncertainty problem can be overcome with a closed-loop response. The controller of the sliding mode technique consists of two components. The first is design equivalent control law to maintain the system state trajectory on the sliding surface. The second is design switching control law to reach the sliding surface. The Lyapunov theorem is used to ensure the stability of the system. Simulation results verify the robustness of the controller.
\end{abstract}

\section{Introduction}

Quadcopter has been applied in many fields such as business [1], agriculture [2], surveillance, and security [4]. Nowadays many methods have been developed to control quadcopter including linear and non-linear controls. Some previous studies were simulation's research of quadcopter controller using PD [5], PID [6] [7] [8] and sliding mode control (SMC) [9]. The PD controller can be reduced the uncertainties of a quadcopter by manually select filter parameters and the results show that the output can follow the reference but there is a steady state error when added disturbance [5]. The PID controller can follow the desired path but level of precision in moving need to be improved [6] [7] [8]. The simulation results of illustrate that proposed SMC controller provides significant reduction of disturbances influence on Quadcopter tracking performances but the gain of switching control could cause high chattering phenomenon if added external disturbance [9]. Based on some literature the SMC control has a robust response, although there is weakness such as high chattering. This weakness can be overcome by adjusting the gain value in the switching control. 
SMC with automatic tuning of gain value on switching control will be investigated on Quadcopter flying. This technique can reduce the effect of the uncertainties and disturbances then minimize the chattering.

\section{Dynamics of a Quadcopter}

The mathematical model of the quadcopter uses 12 states, namely $\mathrm{x}, \mathrm{y}, \mathrm{z}$ as position of quadcopter, $\dot{x}, \dot{y}, \dot{z}$ as velocity of $x, y, z$, then $\phi, \theta, \psi$ as roll, pitch, yaw angle, and $\dot{\phi}, \dot{\theta}, \dot{\psi}$ as angular velocity. The dynamic of system will be represent in state space model.

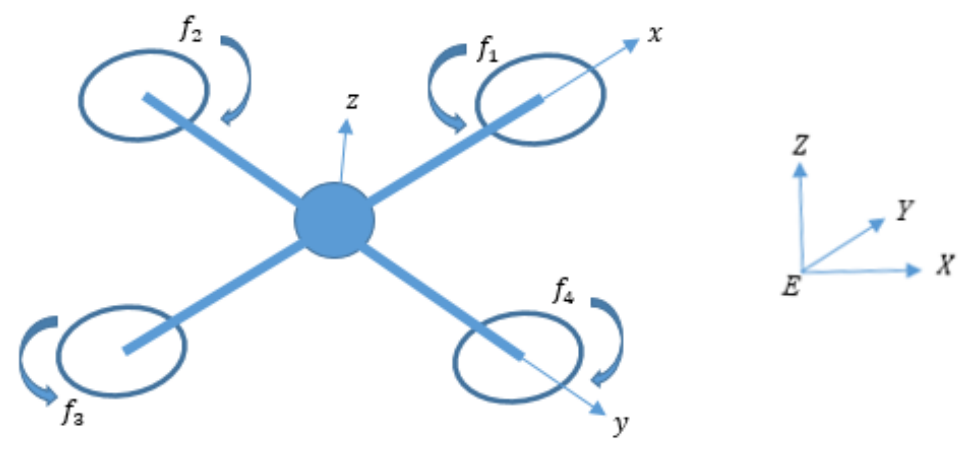

Fig. 1. Model of quadcopter

The Quadopter model is obtained from the Newton-Euler equation. In the first stage, use Newton second law to get the equation of translational motion [10].

$$
\begin{aligned}
& F+d_{F}=m \dot{v}+\omega \times m v, \\
& F_{g}-F_{\text {thrust }}+d_{F}=m \dot{v}+\omega \times m v,
\end{aligned}
$$

where $\boldsymbol{F}_{\boldsymbol{g}}$ is gravity, $\boldsymbol{F}_{\text {thrust }}$ are rotation matrix of vertical thrust $\left[\begin{array}{lll}0 & 0 & \boldsymbol{U}_{\mathbf{1}}\end{array}\right]^{T}, \boldsymbol{d}_{\boldsymbol{F}}=\left[\begin{array}{ll}\boldsymbol{d}_{\boldsymbol{x}} \boldsymbol{d}_{\boldsymbol{y}} \boldsymbol{d}_{\boldsymbol{z}}\end{array}\right]^{T}$ are external disturbances, $m$ is mass of quadcopter, $\boldsymbol{v}=\left[\begin{array}{lll}\dot{\mathrm{x}} & \dot{\mathrm{y}} & \dot{\mathrm{z}}\end{array}\right]^{T}$ are velocity, $\boldsymbol{\omega}=\left[\begin{array}{lll}\dot{\phi} & \dot{\theta} & \dot{\psi}\end{array}\right]^{T}$ are angular velocity.

$$
\left.\left[\begin{array}{c}
0 \\
0 \\
m g
\end{array}\right]-\left(R_{z}(\psi) \cdot R_{y}(\theta) \cdot R_{x}(\phi)\right)\left[\begin{array}{c}
0 \\
0 \\
U_{1}
\end{array}\right]+\left[\begin{array}{c}
d_{x} \\
d_{y} \\
d_{z}
\end{array}\right]=m\left[\begin{array}{c}
\ddot{x} \\
\ddot{y} \\
\ddot{z}
\end{array}\right]+\left[\begin{array}{c}
\dot{\phi} \\
\dot{\theta} \\
\dot{\psi}
\end{array}\right] \times\left[\begin{array}{c}
\dot{x} \\
\dot{y} \\
\dot{z}
\end{array}\right]\right] .
$$

The rotation matrix of roll, pitch, and yaw denoted as : 


$$
\begin{aligned}
R_{z}(\psi) \cdot R_{y}(\theta) \cdot R_{x}(\phi)= & {\left[\begin{array}{ccc}
c_{\psi} & s_{\psi} & 0 \\
-s_{\psi} & c_{\psi} & 0 \\
0 & 0 & 1
\end{array}\right]\left[\begin{array}{ccc}
c_{\theta} & 0 & s_{\theta} \\
0 & 1 & 0 \\
-s_{\theta} & 0 & c_{\theta}
\end{array}\right]\left[\begin{array}{ccc}
1 & 0 & 0 \\
0 & c_{\phi} & s_{\phi} \\
0 & -s_{\phi} & c_{\phi}
\end{array}\right] } \\
& =\left[\begin{array}{ccc}
c_{\theta} c_{\psi} & s_{\phi} s_{\theta} c_{\psi}-c_{\phi} s_{\psi} & s_{\phi} s_{\psi}+c_{\phi} s_{\theta} c_{\psi} \\
c_{\theta} s_{\psi} & s_{\phi} s_{\theta} s_{\psi}+c_{\phi} c_{\psi} & -s_{\phi} c_{\psi}+c_{\phi} s_{\theta} s_{\psi} \\
-s_{\theta} & s_{\phi} c_{\theta} & c_{\phi} c_{\theta}
\end{array}\right]
\end{aligned}
$$

Substituting equation 4 into equation 3

$$
\left[\begin{array}{c}
\ddot{x} \\
\ddot{y} \\
\ddot{z}
\end{array}\right]=\frac{1}{m}\left[\left[\begin{array}{c}
0 \\
0 \\
m g
\end{array}\right]-\left[\begin{array}{ccc}
c_{\theta} c_{\psi} & s_{\phi} s_{\theta} c_{\psi}-c_{\phi} s_{\psi} & s_{\phi} s_{\psi}+c_{\phi} s_{\theta} c_{\psi} \\
c_{\theta} s_{\psi} & s_{\phi} s_{\theta} s_{\psi}+c_{\phi} c_{\psi} & -s_{\phi} c_{\psi}+c_{\phi} s_{\theta} s_{\psi} \\
-s_{\theta} & s_{\phi} c_{\theta} & c_{\phi} c_{\theta}
\end{array}\right]\left[\begin{array}{c}
0 \\
0 \\
U_{1}
\end{array}\right]+\left[\begin{array}{c}
d_{x} \\
d_{y} \\
d_{z}
\end{array}\right]\right]-\left[\begin{array}{c}
\dot{\phi} \\
\dot{\theta} \\
\dot{\psi}
\end{array}\right] \times\left[\begin{array}{c}
\dot{x} \\
\dot{y} \\
\dot{z}
\end{array}\right] .
$$

[5]

Assume that $\boldsymbol{v}=\left[\begin{array}{lll}\dot{\mathrm{x}} & \dot{\mathrm{y}} & \dot{\mathrm{z}}\end{array}\right]^{T}$ and $\boldsymbol{\omega}=\left[\begin{array}{lll}\dot{\phi} & \dot{\theta} & \dot{\psi}\end{array}\right]^{T}$ are equal with 0 , we can find $\dot{\boldsymbol{v}}$

$$
\begin{aligned}
& \ddot{x}=-\frac{1}{m}\left(s_{\phi} s_{\psi}+c_{\phi} s_{\theta} c_{\psi}\right) U_{1}+\frac{1}{m} d_{x}, \\
& \ddot{y}=-\frac{1}{m}\left(-s_{\phi} c_{\psi}+c_{\phi} s_{\theta} s_{\psi}\right) U_{1}+\frac{1}{m} d_{y}, \\
& \ddot{z}=g-\frac{1}{m}\left(c_{\phi} c_{\theta}\right) U_{1}+\frac{1}{m} d_{z} .
\end{aligned}
$$

Rotational Euler equation can be written as :

$$
M_{b}=\omega \times(I \cdot \omega)+I \cdot \dot{\omega}=\left[\begin{array}{l}
U_{2} \\
U_{3} \\
U_{4}
\end{array}\right],
$$

where $\left[\boldsymbol{U}_{\mathbf{2}} \boldsymbol{U}_{\mathbf{3}} \boldsymbol{U}_{\mathbf{4}}\right]^{T}$ are the control torques of roll, pitch, and yaw,

$$
\left[\begin{array}{c}
U_{2} \\
U_{3} \\
U_{4}
\end{array}\right]=\left[\begin{array}{c}
\dot{\phi} \\
\dot{\theta} \\
\dot{\psi}
\end{array}\right] \times\left[\begin{array}{c}
I_{x x} \dot{\phi} \\
I_{y y} \dot{\theta} \\
I_{z z} \dot{\psi}
\end{array}\right]+\left[\begin{array}{c}
I_{x x} \ddot{\phi} \\
I_{y y} \ddot{\theta} \\
I_{z z} \ddot{\psi}
\end{array}\right]
$$

Based on equation 10, we can get :

$$
\begin{aligned}
& U_{2}=\left(I_{z z}-I_{y y}\right) \dot{\boldsymbol{\theta}} \dot{\boldsymbol{\psi}}+I_{x x} \ddot{\boldsymbol{\phi}}, \\
& \boldsymbol{U}_{3}=\left(I_{x x}-I_{z z}\right) \dot{\boldsymbol{\phi}} \dot{\boldsymbol{\psi}}+I_{y y} \ddot{\boldsymbol{\theta}}, \\
& \boldsymbol{U}_{4}=\left(I_{y y}-I_{x x}\right) \dot{\boldsymbol{\phi}} \dot{\boldsymbol{\theta}}+I_{z z} \ddot{\boldsymbol{\psi}} .
\end{aligned}
$$

Finally, the derivatives of the angular velocity can be written as :

$$
\begin{aligned}
& \ddot{\boldsymbol{\phi}}=\frac{U_{2}+\left(I_{y y}-I_{z z}\right) \dot{\theta} \dot{\psi}}{I_{x x}}, \\
& \ddot{\theta}=\frac{U_{3}+\left(I_{z z}-I_{x x}\right) \dot{\phi} \dot{\psi}}{I_{y y}}, \\
& \ddot{\boldsymbol{\psi}}=\frac{U_{4}+\left(I_{x x}-I_{y y}\right) \dot{\phi} \dot{\theta}}{I_{z z}} .
\end{aligned}
$$


The state space of the quadcopter has the following form

$$
\begin{aligned}
& X=\left[\begin{array}{llllllllllll}
x_{1} & x_{2} & x_{3} & x_{4} & x_{5} & x_{6} & x_{7} & x_{8} & x_{9} & x_{10} & x_{11} & x_{12}
\end{array}\right]^{T},
\end{aligned}
$$

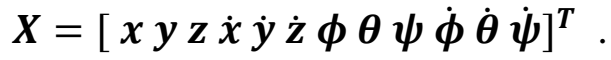

Then, we get

$$
\begin{aligned}
& \dot{x}_{1}=\dot{x}=x_{4}, \\
& \dot{x}_{2}=\dot{y}=x_{5} \\
& \dot{x}_{3}=\dot{z}=x_{6}, \\
& \dot{x}_{4}= \ddot{x}=-\frac{1}{m}\left(s_{\phi} s_{\psi}+c_{\phi} s_{\theta} c_{\psi}\right) U_{1}+\frac{1}{m} d_{x}, \\
& \dot{x}_{5}=\ddot{y}=\ddot{y}=-\frac{1}{m}\left(-s_{\phi} c_{\psi}+c_{\phi} s_{\theta} s_{\psi}\right) U_{1}+\frac{1}{m} d_{y} \\
& \dot{x}_{6}=\ddot{z}=g-\frac{1}{m}\left(c_{\phi} c_{\theta}\right) U_{1}+\frac{1}{m} d_{z} \\
& \dot{x}_{7}=\dot{\phi}=x_{10}, \\
& \dot{x}_{8}=\dot{\theta}=x_{11}, \\
& \dot{x}_{9}=\dot{\psi}=x_{12}, \\
& \dot{x}_{10}=\ddot{\phi}=\frac{U_{2}+\left(I_{y y}-I_{z z}\right) \dot{\theta} \dot{\psi}}{I_{x x}} \\
& \dot{x}_{11}=\ddot{\theta}=\frac{U_{3}+\left(I_{z z}-I_{x x}\right) \dot{\phi} \dot{\psi}}{I_{y y}} \\
& \dot{x}_{12}=\ddot{\psi}=\frac{U_{4}+\left(I_{y y}-I_{x x}\right) \dot{\phi} \dot{\theta}}{I_{z z}}
\end{aligned}
$$

and input control of $\boldsymbol{u}_{\boldsymbol{x}}$ and $\boldsymbol{u}_{\boldsymbol{y}}$ can be represented as

$$
\begin{aligned}
& u_{x}=s_{\phi} s_{\psi}+c_{\phi} s_{\theta} c_{\psi}, \\
& u_{y}=-s_{\phi} c_{\psi}+c_{\phi} s_{\theta} s_{\psi} .
\end{aligned}
$$

The total thrust/force $\boldsymbol{U}_{\mathbf{1}}$, roll torque $\boldsymbol{U}_{\mathbf{2}}$, pitch torque $\boldsymbol{U}_{3}$, and yaw torque $\boldsymbol{U}_{\mathbf{4}}$ can be expressed as follows

$$
\begin{aligned}
& U_{1}=F=f_{1}+f_{2}+f_{3}+f_{4}, \\
& U_{2}=f_{2}-f_{4} \\
& U_{3}=f_{1}-f_{3} \\
& U_{4}=f_{1}+f_{3}-f_{2}-f_{4} .
\end{aligned}
$$

Quadcopter parameters used can be seen in table 1.

Table 1. Parameter of a quadcopter.

\begin{tabular}{|l|l|}
\hline \multicolumn{1}{|c|}{ Parameter } & \multicolumn{1}{c|}{ Value } \\
\hline Distance rotor from centre $(\boldsymbol{l})$ & $0.3 \boldsymbol{m}$ \\
\hline Mass $(\boldsymbol{m})$ & $2.5 \mathrm{~kg}$ \\
\hline Inertial moment on x axis $\left(\boldsymbol{J}_{\boldsymbol{x} \boldsymbol{x}}\right)$ & $0.04 \mathrm{~kg} \cdot \boldsymbol{m}^{2}$ \\
\hline Inertial moment on y axis $\left(\boldsymbol{J}_{\boldsymbol{y y}}\right)$ & $0.04 \mathrm{~kg} \cdot \boldsymbol{m}^{2}$ \\
\hline Inertial moment on z axis $\left(\boldsymbol{J}_{z z}\right)$ & $0.045 \mathrm{~kg} \cdot \boldsymbol{m}^{2}$ \\
\hline Gain $(\boldsymbol{K})$ & $150 \mathrm{rad} / \boldsymbol{s}$ \\
\hline
\end{tabular}




\section{Controller Design}

The SMC control consists of equivalent control and switching control. In the equivalent control for the $\mathrm{Z}$ axis, the first step is designing a sliding surface. The sliding surface is defined as:

$$
\begin{aligned}
& s_{5}=\dot{\tilde{\boldsymbol{n}}}_{5}+\lambda_{5} \widetilde{\boldsymbol{n}}_{5}, \\
& s_{5}=\left(\dot{x}_{5}-\dot{x}_{5 d}\right)+\lambda_{5}\left(x_{5}-x_{5 d}\right),
\end{aligned}
$$

where $\lambda_{5}>0$, then derivative of $\boldsymbol{s}_{\mathbf{5}}$

$$
\begin{aligned}
& \dot{s}_{5}=\ddot{\tilde{\tilde{n}}}_{5}+\lambda_{5} \dot{\tilde{\tilde{n}}}_{5}=0, \\
& 0=\left(\ddot{x}_{5}-\ddot{x}_{5 d}\right)+\lambda_{5}\left(\dot{x}_{5}-\dot{x}_{5 d}\right), \\
& \quad 0=\left(\dot{x}_{6}-\ddot{x}_{5 d}\right)+\lambda_{5}\left(\dot{x}_{5}-\dot{x}_{5 d}\right),
\end{aligned}
$$

$\dot{\boldsymbol{x}}_{\mathbf{6}}$ can be defined as :

$$
\dot{x}_{6}=\ddot{z}=g-\frac{1}{m}\left(c_{\phi} c_{\theta}\right) U_{1}+\frac{1}{m} d_{z} .
$$

Substituting equation (43) to equation (42)

$$
0=\left(g-\frac{1}{m}\left(c_{\phi} c_{\theta}\right) U_{1}+\frac{1}{m} d_{z}-\ddot{x}_{5 d}\right)+\lambda_{5}\left(\dot{x}_{5}-\dot{x}_{5 d}\right)
$$

and we get equivalent control:

$$
U_{\mathrm{eq}}=\frac{m}{\cos x_{7} \cdot \cos x_{8}}\left(g+\frac{1}{m} d_{z}-\ddot{x}_{5 d}\right)+\lambda_{5}\left(\dot{x}_{5}-\dot{x}_{5 d}\right) .
$$

The switching control for $\mathrm{Z}$ axis can be written as:

$$
u_{s}=-K_{5} \operatorname{sign}\left(s_{5}\right) \text {. }
$$

Finally, SMC controller can be obtained by adding equation (45) and (46)

$$
U_{1}=\frac{m}{\cos x_{7} \cdot \cos x_{8}}\left(g+\frac{1}{m} d_{z}-\ddot{x}_{5 d}\right)+\lambda_{5}\left(\dot{x}_{5}-\dot{x}_{5 d}\right)-K_{5} \operatorname{sign}\left(s_{5}\right) .
$$

The stability of the system can be proven using the Lyapunov stability method Lyapunov function is defined as:

$$
V=\frac{1}{2} S^{2}>0
$$

and

$$
\begin{aligned}
\dot{V} & =s \dot{s}<0 \\
& =\left(\left(\dot{x}_{5}-\dot{x}_{5 d}\right)+\lambda_{5}\left(\boldsymbol{x}_{5}-\boldsymbol{x}_{5 d}\right)\right)\left(\left(\ddot{x}_{5}-\ddot{x}_{5 d}\right)+\lambda_{5}\left(\dot{x}_{5}-\dot{x}_{5 d}\right)\right) .
\end{aligned}
$$

Subtituting equation (43) into equation (49):

$\dot{V}=\left(\left(\dot{x}_{5}-\dot{x}_{5 d}\right)+\lambda_{5}\left(x_{5}-x_{5 d}\right)\right)\left(\left(g-\frac{1}{m}\left(c_{\phi} c_{\theta}\right) U_{1}+\frac{1}{m} d_{z}-\ddot{x}_{5 d}\right)+\lambda_{5}\left(\dot{x}_{5}-\dot{x}_{5 d}\right)\right)$.

Then, substituting equation (47) into equation (50): 


$$
\begin{aligned}
\dot{V}= & \left(\left(\dot{x}_{5}-\dot{x}_{5 d}\right)+\lambda_{5}\left(x_{5}-x_{5 d}\right)\right)\left(\left(g-\frac{1}{m}\left(c_{\phi} c_{\theta}\right)\left(\frac{m}{\cos x_{7} \cdot \cos x_{8}}\left(g+\frac{1}{m} d_{z}-\ddot{x}_{5 d}\right)+\right.\right.\right. \\
& \left.\left.\left.\lambda_{5}\left(\dot{x}_{5}-\dot{x}_{5 d}\right)-K_{5} \operatorname{sign}\left(s_{5}\right)\right)+\frac{1}{m} d_{z}-\ddot{x}_{5 d}\right)+\lambda_{5}\left(\dot{x}_{5}-\dot{x}_{5 d}\right)\right) .
\end{aligned}
$$

We can eliminated some equation in equation (51) and choose $\mathrm{K}$ to be minus large enough to get stable in the sense of Lyapunov. K gain can change when the system gets disturbances. The equation can be seen in (52)

where $\boldsymbol{\varepsilon}_{\mathbf{5}}$ is defined as:

$$
\widehat{K}_{5}=-\mu_{5} m \operatorname{Sign}\left(s_{5}\right)\left(-\varepsilon_{5}\right)
$$

$$
\varepsilon_{5}=m\left(\dot{x}_{6}+g-K_{5} \operatorname{sign}\left(s_{5}\right)\right)-U_{1}
$$

Then, control for $\mathrm{Z}$ axis can be written as:

$$
U_{1}=\frac{m}{\cos x_{7} \cdot \cos x_{8}}\left(g+\frac{1}{m} d_{z}-\ddot{x}_{5 d}\right)+\lambda_{5}\left(\dot{x}_{5}-\dot{x}_{5 d}\right)-\widehat{K}_{5} \operatorname{sign}\left(s_{5}\right)
$$

with same technique we get input control

$$
\begin{gathered}
u_{x}=\left(\frac{d_{x}}{m}+\ddot{x}_{3 d}-\left(\lambda_{3}\left(\dot{x}_{3}-\dot{x}_{3 d}\right)\right)\right) \frac{m}{U_{1}}-\widehat{K}_{3} \operatorname{sign}\left(s_{3}\right), \\
u_{y}=\left(\frac{d_{y}}{m}+\ddot{x}_{4 d}-\lambda_{4}\left(\dot{x}_{4}-\dot{x}_{4 d}\right)\right) \frac{m}{U_{1}}-\widehat{K}_{4} \operatorname{sign}\left(s_{4}\right), \\
U_{2}=\left(I_{z z}-I_{y y}\right) x_{11} x_{12}+\left(\ddot{x}_{9 d}-\lambda_{9}\left(\dot{x}_{9}-\dot{x}_{9 d}\right)\right) I_{x x}-K_{9} \operatorname{sign}\left(s_{9}\right), \\
U_{3}=\left(I_{x x}-I_{z z}\right) x_{10} x_{12}+\left(\ddot{x}_{10 d}-\lambda_{10}\left(\dot{x}_{10}-\dot{x}_{10 d}\right)\right) I_{y y}-K_{10} \operatorname{sign}\left(s_{10}\right), \\
U_{4}=\left(I_{y y}-I_{x x}\right) x_{10} x_{11}+I_{z z}\left(\ddot{x}_{11 d}-\lambda_{11}\left(\dot{x}_{11}-\dot{x}_{11 d}\right)\right)-K_{11} \operatorname{sign}\left(s_{11}\right),
\end{gathered}
$$

\section{Simulation Results}

In Figure 2 shows the quadcopter can follow the set point without steady state error and requires a rise time of 8 seconds to reach the $\mathrm{x}$-axis change as far as 5 meters. The output response in Figures 3 and 4 shows good performance despite being given payload as disturbance (Figure 8). Then in Figures 5 and 6 shows the response of the Roll angle and Pitch angle to the reference signal has fast rise time. Figure 7 shows that the gain in switching controls can change automatically adjusting changes in dynamics of the system due to disturbance and uncertainty. When given a load as a disturbance (Figure 8), the gain value at $\mathrm{Kz}$ increases so that the quadcopter can maintain its position on the $\mathrm{Z}$ axis. 


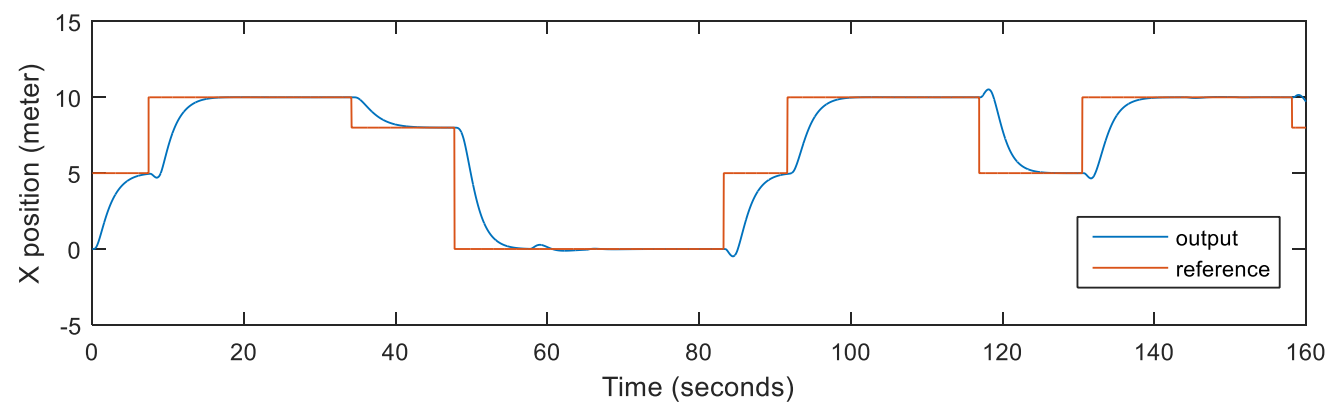

Figure 2 Position on $\mathrm{X}$ axis

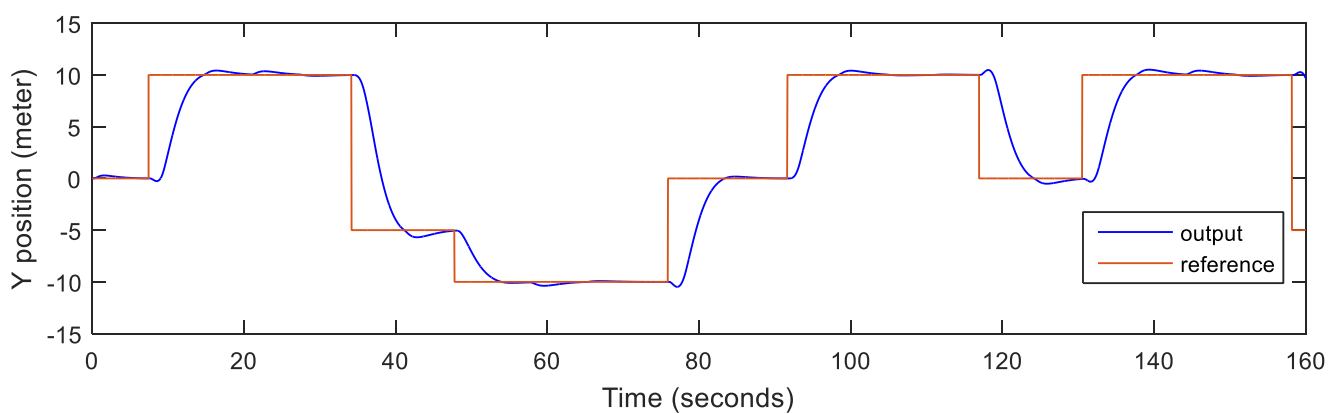

Figure 3 Position on $\mathrm{Y}$ axis

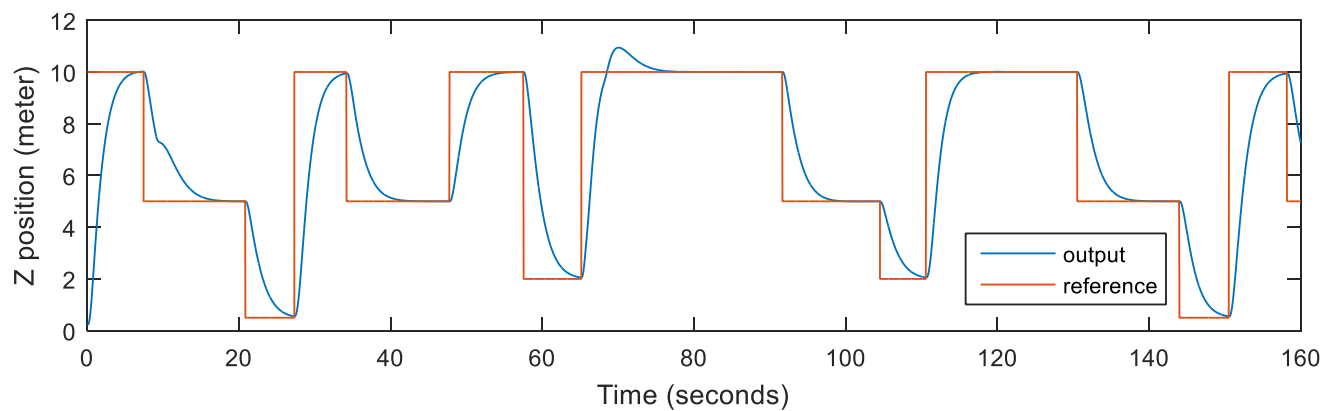

Figure 4 Position on $\mathrm{Z}$ axis

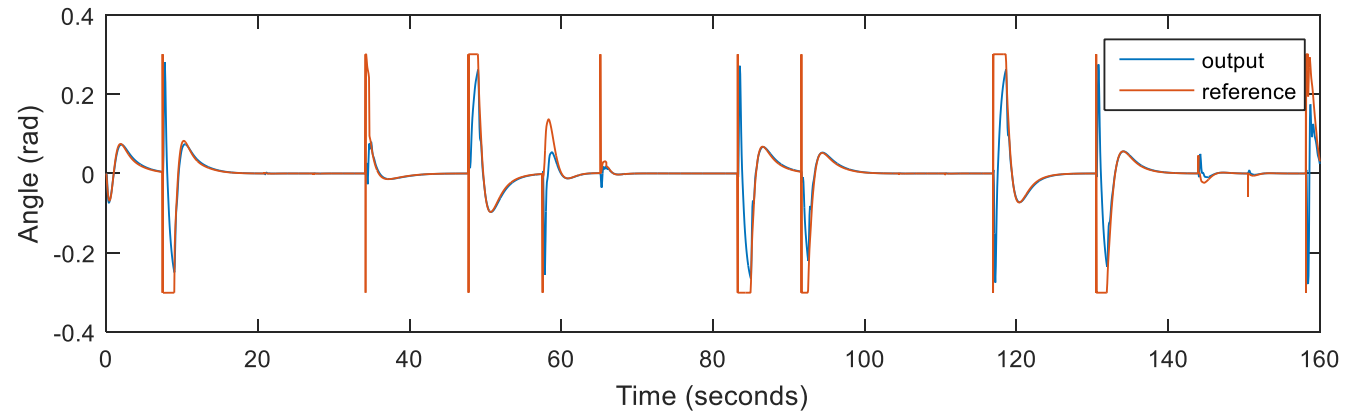

Figure 5 Roll angle 


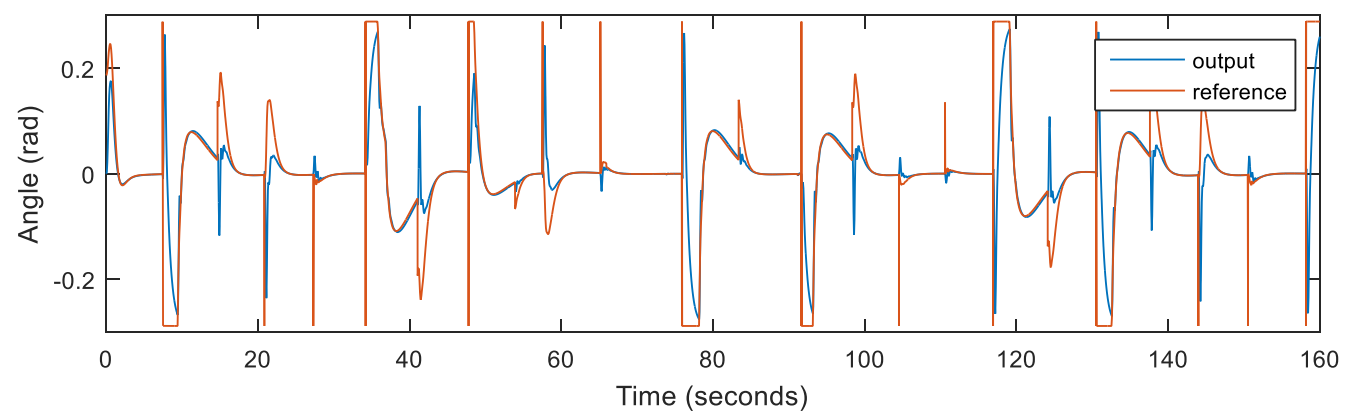

Figure 6 Pitch angle

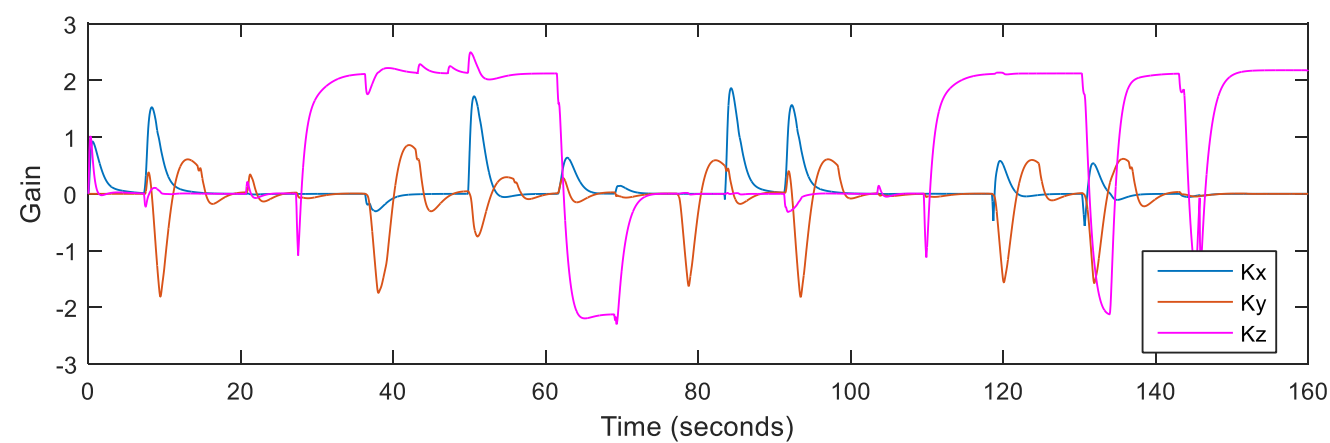

Figure 7 Gain change in simulation

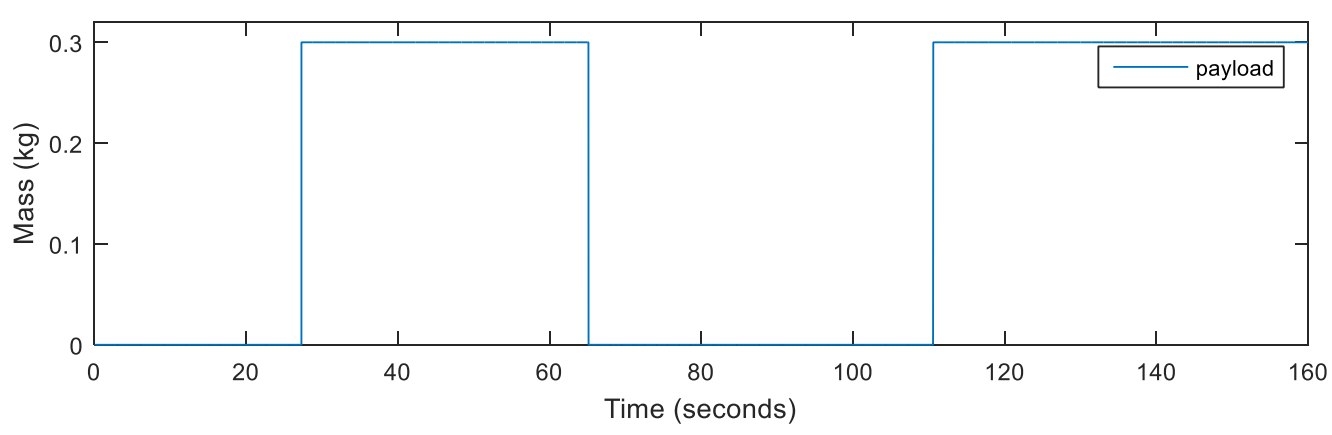

Figure 8 Payload as disturbance

\section{Conclusion}

The simulation results show that the SMC can control the quadcopter following the reference of position without causing a chattering phenomenon, then the switching control can change automatically when there are changes in parameters and disturbances.

\section{References}

[1] A. M. Lawson, "Consumer Behaviors of Drone Delivery," Bachelor Theses, Appalachian State University, 2017.

[2] L. P. Hafsal, "Precision Agriculture with Unmaned Aerial Vehicles for SMC estimation - Towards a more Sustainable Agriculture," Master Thesis, Hedmark University of Applied Sciences, 2016.

[3] N. H. Motlagh, M. Bagaa, and T. Taleb, "UAV-Based IoT Platform: A Crowd Surveillance use Case," IEEE Communications Magazine, vol. 55, pp. 128-134, February 2017. 
[4] D. He, S. Chan, and M. Guizani, "Drone-Assisted Public Safety Networks: The Security Aspect," IEEE Communications Maagazine, vol. 55, pp. 218-233, April 2017.

[5] G. Shincy and E. Varghese, "Design and Simulation of Robust Filter for Tracking Control of Quadcopter System," IEEE International Conference on circuits Power and Computing Technologies, Apr. 2017.

[6] R. A. Garcia, F. R. Rubio, and M. G. Ortega,"Robust PID Control of the Quadrotor Helicopter," IFAC Conference, Mar. 2012.

[7] S. Abdelmoeti and R. Carloni, "Robust Control of an Unmanned Aerial Vehicle Using the Parameter Space Approach," Project on Robotics and Mechatronics at University of Twente.

[8] B. J. Emran, J. Dias, L. Seneviratne, and G. Cai, "Robust Adaptive Design for Quadcopter Payload Add and Drop Applications," Chinese control conference, pp. 3252-3257, July 2015.

[9] Stevanovic, Kasac, and Stepanic,'Robust Tracking Control of a Quadrotor Helicopter without Velocity Measurement," International DAAAM Symponsium, vol. 23, pp. 595-600, 2012.

[10] P. Pounds, R. Mahony, and P. Corke, "Modelling and control of a quad-rotor robot," in Australasian Conference on Robotics and Automation 2006. Auckland, New Zealand: Australian Robotics and Automation Association Inc., 2006. 